\title{
Insegurança alimentar no Brasil segundo diferentes cenários sociodemográficos
}

\author{
Food insecurity in Brazil \\ in accordance with different socio-demographic scenarios
}

Thaíse Alves Bezerra ${ }^{1}$

Ricardo Alves de Olinda ${ }^{2}$ Dixis Figueroa Pedraza ${ }^{1}$
${ }^{1}$ Programa de Pós-

Graduação em Saúde

Pública, Universidade

Estadual da Paraíba. Av. das

Baraúnas 351, Bodocongó.

58109-753 Campina

Grande PB Brasil.

thaise_gba@hotmail.com

${ }^{2}$ Departamento de

Estatística, Universidade

Estadual da Paraíba.

Campina Grande PB Brasil.
Abstract The scope of this article is to determine the prevalence of food insecurity, as well as associated factors in different socio-demographic contexts in Brazil. A systematic review with meta-analysis of articles published from 2004 to 2013 on Food and Nutrition Security based on searches in databases. The articles were categorized in accordance with the origin of their samples, duly calculating the weighted average prevalence of food insecurity and odds ratios. The weighted averages of the prevalence of food insecurity for schools/ daycares, health services/beneficiaries of the Bolsa Família Program and socially-challenged population groups were $1.8 \%, 76.6 \%, 87.2 \%$ and 25.9 $\%$, respectively, with higher odds ratios in relation to population-based studies and results of the $\mathrm{Na}$ tional Household Sample Survey (PNAD). Family income, number of members in the household and the type of housing were the main variables that revealed a significant inverse relationship with food insecurity. The results show the social determinants of food insecurity in Brazil.

Key words Food and Nutrition Security, Systematic review, Poverty
Resumo O objetivo deste artigo é apontar a prevalência de insegurança alimentar, assim como fatores associados, em diferentes cenários sociodemográficos no Brasil. Realizou-se revisão sistemática com metanálise de artigos publicados de 2004 a 2013 sobre Segurança Alimentar e Nutricional a partir da busca em bases de dados. Calculou-se a média ponderada da prevalência de insegurança alimentar e as Razões de Chances considerando a categorização dos artigos segundo a origem de suas amostras. A média ponderada da prevalência de insegurança alimentar para escolas/creches, serviços de saúde/beneficiários do Programa Bolsa Família e populações em iniquidades sociais foi, respectivamente, de 61,8\%, 76,6\% e 87,2\%, com maiores Razões de Chances em relação aos estudos de base populacional e aos resultados da PNAD. A renda familiar, a quantidade de indivíduos no domicílio e o tipo de moradia foram as principais variáveis que apresentaram relação inversa significante com a insegurança alimentar. Os resultados evidenciam a determinação social da insegurança alimentar no Brasil.

Palavras-chave Segurança alimentar e nutricional, Revisão sistemática, Pobreza 


\section{Introdução}

O conceito de Segurança Alimentar e Nutricional (SAN) adotado no Brasil na Segunda Conferência Nacional de Segurança Alimentar e Nutricional define a SAN como a "realização do direito de todos ao acesso regular e permanente a alimentos de qualidade, em quantidade suficiente, sem comprometer o acesso a outras necessidades essenciais, tendo como base práticas alimentares promotoras de saúde, que respeitem a diversidade cultural e que sejam ambiental, econômica e socialmente sustentáveis"'. Assim, admite-se que a insegurança alimentar no Brasil esteja condicionada pela falta de acesso à alimentação, fator este que depende, predominantemente, da relação entre a renda e o preço dos alimentos ${ }^{2}$.

A atenção permanente à importância da SAN, no Brasil e no mundo, nas interfaces nutrição - saúde - ciências sociais tornou relevante a busca por indicadores capazes de acompanhar a sua evolução, avaliar o impacto das intervenções e estabelecer comparações tempo-espacial ${ }^{3}$. No Brasil, pesquisadores de várias instituições validaram um método para a avaliação direta da SAN, denominada Escala Brasileira de Insegurança Alimentar (EBIA) ${ }^{4}$. A validação da EBIA possibilitou contemplar as especificidades e as diversidades nacionais, tornando-se um instrumento aplicável tanto nas zonas rurais como nas urbanas. 5 . Essa escala tem sido reconhecida como indicador sensível para detectar famílias em risco de insegurança alimentar ${ }^{6}$ e como um instrumento relevante para avaliar os efeitos das políticas públicas ${ }^{5}$.

Apesar de estudos mostrarem associação da insegurança alimentar com as condições sociais e econômicas, sua prevalência no Brasil apresentou proporção maior em relação a outros problemas nutricionais como a desnutrição, com os quais podem não apresentar correlação $0^{7,8}$. Assim, a SAN deve ser considerada como medida importante para um melhor entendimento dos problemas de alimentação e nutrição que deve acompanhar a avaliação específica do estado nutricional ${ }^{9}$.

Em virtude dos argumentos anteriores e das consequências da insegurança alimentar e nutricional, este estudo teve por objetivo apontar a prevalência de insegurança alimentar, assim como fatores associados, em diferentes cenários sociodemográficos no Brasil.

\section{Métodos}

O estudo é uma revisão sistemática com metanálise de artigos científicos sobre SAN desenvolvidos no Brasil com a aplicação da EBIA. A identificação dos artigos de interesse foi realizada em 04 de janeiro de 2014, utilizando-se as bases de dados SciELO (Scientific Electronic Library Online), Lilacs (Literatura Latino-americana e do Caribe em Ciências da Saúde) e PubMed (National Library of Medicine, Bethesda, MD), a partir da palavra-chave pré-definida "Segurança Alimentar e Nutricional". No caso do Pubmed foi acrescida a palavra-chave "Brazil". A busca foi realizada nos idiomas português, inglês e espanhol, considerando artigos publicados entre o período de 2004 a 2013. Optou-se por estudos realizados a partir de 2004, pois a partir deste ano a EBIA ganhou maior visibilidade ao ser incorporada à Pesquisa Nacional por Amostra de Domicílios (PNAD), logo depois da sua validação neste mesmo ano.

Numa segunda etapa, foram aplicados os critérios de exclusão por meio da leitura dos títulos e resumos dos artigos, considerando inadequados para os objetivos propostos as pesquisas de avaliação de alimentos/sanitária, os estudos com animais, os artigos de revisão/reflexão/discussão teórica, aqueles que não utilizaram a EBIA e os que não descreveram a prevalência de insegurança alimentar.

As listas de referências dos estudos selecionados foram utilizadas com o intuito de localizar artigos que não tivessem sido encontrados nas bases de dados. Esses artigos foram submetidos da mesma maneira aos critérios de inclusão e exclusão.

Os artigos incluídos foram submetidos à leitura e à sistematização dos resultados e, em seguida, agrupados em quatro categorias conforme a natureza de obtenção de suas respectivas amostras: escolas/creches, serviços de saúde/beneficiários do Programa Bolsa Família, populações em iniquidades sociais (comunidades indígenas, clientela da Pastoral da Criança, áreas de vulnerabilidade socioeconômica) e, por fim, estudos de base populacional representativos de cidades, regiões ou estados brasileiros.

Foram calculadas para cada uma dessas quatro categorias a média ponderada da prevalência, com respectivas amplitudes, de insegurança alimentar, insegurança alimentar leve, insegurança alimentar moderada e insegurança alimentar grave pelos respectivos tamanhos amostrais. Calcularam-se, ainda, para os três primeiros cenários, a Razão de Chances (RC) dos desfechos 
anteriores para um intervalo de confiança de 95\% (IC95\%), assumindo como prevalências de referência as encontradas nos estudos representativos de cidades, regiões ou estados brasileiros e na PNAD de $2009^{10}$. A significância das diferenças $(p<0,05)$ entre as frequências encontradas foi verificada por meio do teste Qui-quadrado.

\section{Resultados}

Foram identificados 546 estudos. Após a aplicação dos critérios de exclusão, 27 artigos foram selecionados. Foram acrescidos quatro artigos a partir das listas de referências, totalizando 31 que tiveram seus resultados sistematizados. Desses, quatro envolveram amostras obtidas em creches/ escolas, seis foram com amostras procedentes de serviços de saúde/beneficiários do Programa Bolsa Família, quatro foram realizados com populações em iniquidade social e 17 foram classificados como de base populacional. O fluxo do número de estudos incluídos encontra-se na Figura 1.

Nos estudos com amostras obtidas em escolas/creches ${ }^{11-14}$, a insegurança alimentar (IA) oscilou de $46,6 \%{ }^{14}$ a $64,5 \%{ }^{12}$ com média ponderada de $61,8 \%$. A insegurança alimentar leve (IAL), a moderada (IAM) e a grave (IAG) apresentaram médias ponderadas de $39,5 \%, 16,2 \%$ e 7,5\%, respectivamente. No que se refere aos estudos com amostras obtidas em serviços de saúde/beneficiários do Programa Bolsa Família ${ }^{15-20}$, a oscilação de IA foi de $72,0 \%{ }^{17}$ a $88,0 \%{ }^{20}$ e média ponderada de $76,6 \%$. Para os diferentes graus de IA as médias ponderadas foram de 45,0\%, 20,3\% e 11,3\% para IAL, IAM e IAG, respectivamente (Tabela 1).

Naqueles estudos com amostras obtidas em populações em iniquidades sociais ${ }^{21-24}$, a maior prevalência de IA foi $88,2 \%{ }^{21}$, em município de Pernambuco, e a menor de 75,5\% ${ }^{23}$, em municípios de Mato Grosso do Sul. As médias ponderadas de IA, IAL, IAM e IAG foram de 87,2\%, 23,2\%, $35,8 \%$ e $28,1 \%$, respectivamente (Tabela 2 ).

Em relação aos estudos de base populacional representativos de cidades, regiões ou estados brasileiros $^{25-41}$ encontrou-se que a IA oscilou de $23,8 \%{ }^{30}$ a $72,0 \%{ }^{35}$. As médias ponderadas nesta categoria foram de $25,9 \%, 12,9 \%, 8,6 \%$ e $4,2 \%$ para IA, IAL, IAM e IAG, respectivamente (Tabela 3).

Em geral, diversas variáveis demográficas e socioeconômicas foram analisadas pelos pesquisadores nos seus estudos. Em termos proporcionais, considerando a relação entre a quantidade de estudos que consideraram uma determinada

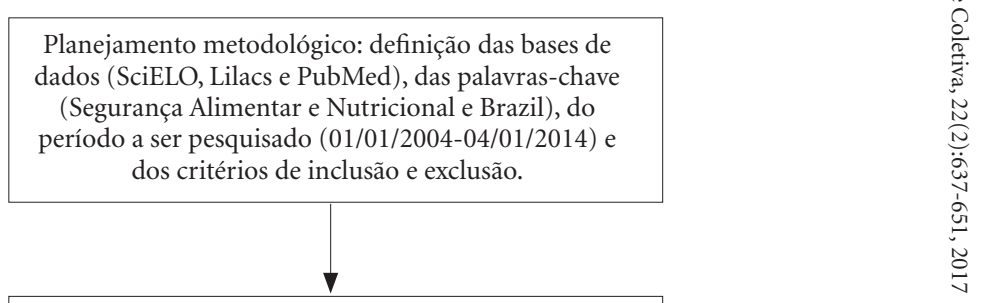

Identificação dos estudos, segundo os critérios de busca pré-estabelecidos: 546 estudos identificados - SciELO: 72

- Lilacs: 260

- PubMed: 223

- Duplicata: 8

- Triplicata: 1

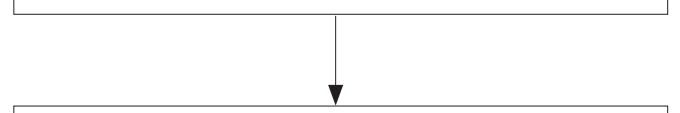

Identificação dos estudos, segundo os critérios de busca pré-estabelecidos: 546 estudos identificados

- SciELO: 72

- Lilacs: 260

- PubMed: 223

- Duplicata: 8

- Triplicata: 1

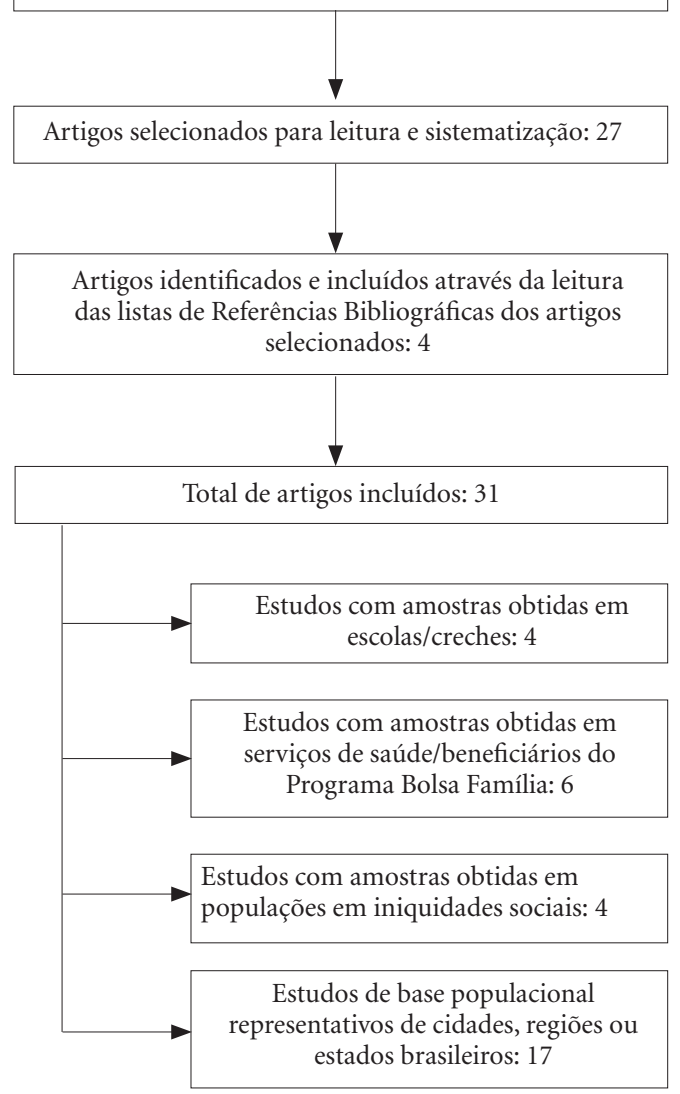

Figura 1. Fluxograma utilizado para a sistematização de estudos sobre Segurança Alimentar e Nutricional realizados no Brasil com a utilização da Escala Brasileira de Insegurança Alimentar (2004-2013). 
Tabela 1. Prevalências de insegurança alimentar, segundo estudos publicados no período de 2004-2013, envolvendo amostras obtidas em instituições (escolas/creches e serviços de saúde/beneficiários do Programa Bolsa Família), no Brasil.

\begin{tabular}{|c|c|c|c|c|c|c|c|}
\hline \multirow[t]{2}{*}{ Fonte } & \multirow[t]{2}{*}{ Local de estudo } & \multirow[t]{2}{*}{$\begin{array}{l}\text { Tamanho } \\
\text { amostral }\end{array}$} & \multicolumn{4}{|c|}{$\begin{array}{c}\text { Prevalências de } \\
\text { Insegurança Alimentar } \\
(\%)\end{array}$} & \multirow[t]{2}{*}{ Associações estatísticas } \\
\hline & & & IA & IAL & IAM & IAG & \\
\hline \multicolumn{8}{|c|}{ Amostras obtidas em escolas/creches } \\
\hline $\begin{array}{l}\text { Figueroa } \\
\text { Pedraza et } \\
\text { al. }^{11}\end{array}$ & $\begin{array}{l}\text { Creches públicas do } \\
\text { estado da Paraíba }\end{array}$ & 332 & 62,0 & 33,4 & 16,0 & 11,7 & $\begin{array}{l}\text { i) Análise bivariada } \\
\text { - Idade da mãe }<20 \text { anos } \\
\text { - Tipo de moradia invadida } \\
\text { - Menor renda familiar per capita } \\
\text { (SM) } \\
\text { - Participar do Programa Bolsa } \\
\text { Família } \\
\text { ii) Análise multivariada } \\
\text { - Menor renda familiar per capita } \\
\text { (SM) }\end{array}$ \\
\hline $\begin{array}{l}\text { Ribeiro-Silva } \\
\text { et al. }{ }^{12}\end{array}$ & $\begin{array}{l}\text { Escolas públicas de } \\
\text { São Francisco do } \\
\text { Conde (BA) }\end{array}$ & 1307 & 64,5 & 42,5 & 15,9 & 6,1 & - Não foi encontrada associação \\
\hline Souza et al. ${ }^{13}$ & $\begin{array}{l}\text { Creches públicas } \\
\text { estaduais de João } \\
\text { Pessoa (PB) }\end{array}$ & 250 & 59,6 & 32,4 & 18,0 & 9,2 & - \\
\hline Vieira et al. ${ }^{14}$ & $\begin{array}{l}\text { Duas escolas públicas } \\
\text { de São Paulo (SP) }\end{array}$ & 195 & 46,6 & - & - & - & - Não foi encontrada associação \\
\hline $\begin{array}{l}\text { Média ponder } \\
\text { pelo tamanho }\end{array}$ & $\begin{array}{l}\text { rada } \\
\text { amostral }\end{array}$ & 2084 & 61,8 & 39,5 & 16,2 & 7,5 & \\
\hline \multicolumn{8}{|c|}{ Amostras obtidas em serviços de saúde/beneficiários do Programa Bolsa Família } \\
\hline Souza et al. ${ }^{15}$ & $\begin{array}{l}\text { Famílias beneficiárias } \\
\text { do Programa Bolsa } \\
\text { Família de Viçosa } \\
(\mathrm{MG})\end{array}$ & 243 & 72,8 & 47,3 & 10,7 & 14,8 & $\begin{array}{l}\text { Análise bivariada } \\
\text { - Escolaridade materna inferior a } \\
\text { dez anos } \\
\text { - Domicílios com quatro ou mais } \\
\text { indivíduos } \\
\text { - Presença de água tratada por } \\
\text { filtração no domicílio } \\
\text { - Classe econômica da ABEP (E) }\end{array}$ \\
\hline Silva et al. ${ }^{16}$ & $\begin{array}{l}\text { Famílias beneficiárias } \\
\text { do Programa Bolsa } \\
\text { Família de São José } \\
\text { dos Ramos (PB) }\end{array}$ & 177 & 76,2 & - & - & - & - Não foi encontrada associação \\
\hline Dias et al. ${ }^{17}$ & $\begin{array}{l}\text { Famílias beneficiárias } \\
\text { do Programa Bolsa } \\
\text { Família e Cesta } \\
\text { Cheia da Unidade } \\
\text { de Saúde da Família } \\
\text { São João Baptista } \\
\text { do município de } \\
\text { Petrópolis (RJ) }\end{array}$ & 172 & 72,0 & 45,3 & 14,5 & 12,2 & - \\
\hline
\end{tabular}

continua

variável e os que indicaram associação com a insegurança alimentar, a pior situação relacionada à renda familiar foi a que apresentou maior proporção de associação (64,0\% dos estudos), em 16 estudos $^{11,20,23,25-29,32,33,35-37,39-41}$ de um total de $25^{11,13,17-29,32-41}$. A seguir, a escolaridade do chefe do domicílio esteve associada à insegurança alimentar em seis estudos ${ }^{20,26,27,35,37,40}$ de um total de 
Tabela 1. continuação

\begin{tabular}{|c|c|c|c|c|c|c|c|}
\hline \multirow[t]{2}{*}{ Fonte } & \multirow[t]{2}{*}{ Local de estudo } & \multirow[t]{2}{*}{$\begin{array}{l}\text { Tamanho } \\
\text { amostral }\end{array}$} & \multicolumn{4}{|c|}{$\begin{array}{c}\text { Prevalências de } \\
\text { Insegurança Alimentar } \\
(\%)\end{array}$} & \multirow[t]{2}{*}{ Associações estatísticas } \\
\hline & & & IA & IAL & IAM & IAG & \\
\hline $\begin{array}{l}\text { Anschau et } \\
\text { al. }{ }^{18}\end{array}$ & $\begin{array}{l}\text { Famílias beneficiárias } \\
\text { de Programa Bolsa } \\
\text { Família de Toledo } \\
(\mathrm{PR})\end{array}$ & 421 & 74,6 & 44,9 & 23,8 & 5,9 & $\begin{array}{l}\text { i) Análise bivariada } \\
\text { - Trabalho informal ou desemprego } \\
\text { do chefe do domicílio } \\
\text { - Presença de indivíduo menor de } \\
18 \text { anos no domicílio } \\
\text { - Domicílios com } 7 \text { ou mais } \\
\text { indivíduos } \\
\text { - Classe econômica da ABEP (D } \\
\text { ou E) } \\
\text { ii) Regressão logística multivariada } \\
\text { - Trabalho informal ou desemprego } \\
\text { do chefe do domicílio } \\
\text { - Domicílios com mais de } 5 \\
\text { indivíduos } \\
\text { - Classe econômica da ABEP (D } \\
\text { ou E) }\end{array}$ \\
\hline $\begin{array}{l}\text { Magrani et } \\
\text { al. }{ }^{19}\end{array}$ & $\begin{array}{l}\text { Famílias beneficiárias } \\
\text { do Programa Bolsa } \\
\text { Família e Cesta Cheia } \\
\text { da Unidade de Saúde } \\
\text { da Família Vila } \\
\text { Felipe do município } \\
\text { Petrópolis (RJ) }\end{array}$ & 274 & 78,3 & 50,4 & 21,3 & 6,6 & - \\
\hline Aires et al. ${ }^{20}$ & $\begin{array}{l}\text { Um Centro de } \\
\text { Saúde da Família de } \\
\text { Maranguape (CE) }\end{array}$ & 200 & 88,0 & 35,0 & 28,5 & 24,5 & $\begin{array}{l}\text { Análise bivariada } \\
\text { - Menor escolaridade do chefe do } \\
\text { domicílio } \\
\text { - Maior número de indivíduos no } \\
\text { domicílio } \\
\text { - Menor renda familiar }\end{array}$ \\
\hline \multicolumn{2}{|c|}{$\begin{array}{l}\text { Média ponderada } \\
\text { pelo tamanho amostral }\end{array}$} & 1487 & 76,6 & 45,0 & 20,3 & 11,3 & \\
\hline
\end{tabular}

IA: Insegurança Alimentar; IAL: Insegurança Alimentar Leve; IAM: Insegurança Alimentar Moderada; IAG: Insegurança Alimentar Grave; SM: Salário Mínimo; ABEP: Associação Brasileira de Empresas de Pesquisa; ABIPEME: Associação Brasileira de Institutos de Pesquisa de Mercado. Observações: i) Figueroa Pedraza et al. ${ }^{11}$, Ribeiro-Silva et al. ${ }^{12}$, e Vieira et al. ${ }^{14}$ utilizaram outras variáveis explanatórias. Nesses estudos, amamentação total $<24$ meses $^{11}$, amamentação exclusiva $<6$ meses $^{11}$, asma ${ }^{12}$ e desnutrição infantil ${ }^{14}$ representaram fatores associados à insegurança alimentar. ii) Silva et al. ${ }^{16}$ utilizaram outras variáveis explanatórias. Nesse estudo, o consumo alimentar inadequado da família representou um fator associado à insegurança alimentar: Dado indisponível no manuscrito ou análise não realizada.

dez $\mathrm{z}^{18-20,24,26,27,34,35,37,40}$ que utilizaram essa variável, ou seja, uma proporção de $60,0 \%$ dos estudos, sendo sempre as famílias com pior indicador as mais vulneráveis a estados de insegurança alimentar.

As proporções de estudos que encontraram associação à insegurança alimentar também foram relevantes para as variáveis: i) região geográfica, com pior situação para a zona rural, em quatro estudos ${ }^{27-29,32}$ de sete s-33 $^{27}$ que investigaram essa variável (57,1\% dos estudos); ii) número de indivíduos no domicílio, sendo maior a probabilidade de insegurança alimentar entre as famílias com maior quantidade de indivíduos, em 10 estudos $^{15,18,20,23,25-27,29,35,40}$ de um total de $21^{11-20,23-}$ 27,29,35,36,39-41 (47,6\% dos estudos); iii) tipo de moradia, em três ${ }^{11,37,39}$ de sete estudos ${ }^{11,13,16,26,37,39,41}$ (42,8\% dos estudos) que indicaram pior situação nos casos de moradia precária; iv) participação em programas de transferência de renda e/ou 
Tabela 2. Prevalências de insegurança alimentar, segundo estudos publicados no período de 2004-2013, envolvendo amostras obtidas em populações em iniquidades sociais, no Brasil.

\begin{tabular}{|c|c|c|c|c|c|c|c|}
\hline \multirow[t]{2}{*}{ Fonte } & \multirow[t]{2}{*}{ Local de estudo } & \multirow{2}{*}{$\begin{array}{l}\text { Tamanho } \\
\text { amostral }\end{array}$} & \multicolumn{4}{|c|}{$\begin{array}{c}\text { Prevalências de } \\
\text { Insegurança Alimentar (\%) }\end{array}$} & \multirow[t]{2}{*}{ Associações estatísticas } \\
\hline & & & IA & IAL & IAM & IAG & \\
\hline $\begin{array}{l}\text { Oliveira et } \\
\text { al. }^{21}\end{array}$ & $\begin{array}{l}\text { Famílias residentes } \\
\text { em áreas de condições } \\
\text { socioeconômicas } \\
\text { precárias de Gameleira } \\
\text { (PE) }\end{array}$ & 501 & 88,2 & 17,8 & 33,5 & 36,9 & $\begin{array}{l}\text { - Não foi encontrada } \\
\text { associação }\end{array}$ \\
\hline $\begin{array}{l}\text { Oliveira et } \\
\text { al. }^{22}\end{array}$ & $\begin{array}{l}\text { Localidade com } \\
\text { Baixo Índice de } \\
\text { Desenvolvimento } \\
\text { Humano, São João do } \\
\text { Tigre (PB) }\end{array}$ & 458 & 87,3 & 25,5 & 40,2 & 21,6 & $\begin{array}{l}\text { - Não foi encontrada } \\
\text { associação }\end{array}$ \\
\hline $\begin{array}{l}\text { Fávaro et } \\
\text { al. }^{23}\end{array}$ & $\begin{array}{l}\text { Aldeias Olho D’Água, } \\
\text { Água Azul e Oliveiras } \\
\text { dos municípios Dois } \\
\text { Irmãos do Buriti e } \\
\text { Sidrolândia (MS) }\end{array}$ & 49 & 75,5 & 22,4 & 32,7 & 20,4 & $\begin{array}{l}\text { Análise bivariada } \\
\text { - Menor escolaridade da mãe } \\
\text { - Maior número de filhos por } \\
\text { grupo familiar } \\
\text { - Maior número de } \\
\text { indivíduos no domicílio } \\
\text { - Menor renda familiar per } \\
\text { capita (SM) }\end{array}$ \\
\hline $\begin{array}{l}\text { Pereira et } \\
\text { al. }^{24}\end{array}$ & $\begin{array}{l}\text { Clientela da Pastoral } \\
\text { da Criança em região } \\
\text { de alta vulnerabilidade } \\
\text { social de São Paulo } \\
\text { (SP) }\end{array}$ & 83 & 88,0 & 44,6 & 27,7 & 15,7 & - \\
\hline \multicolumn{2}{|c|}{$\begin{array}{l}\text { Média ponderada pelo tamanho } \\
\text { amostral }\end{array}$} & 1091 & 87,2 & 23,2 & 35,8 & 28,1 & \\
\hline
\end{tabular}

IA: Insegurança Alimentar; IAL: Insegurança Alimentar Leve; IAM: Insegurança Alimentar Moderada; IAG: Insegurança Alimentar Grave; SM: Salário Mínimo. Observações: Oliveira et al. ${ }^{21}$, Oliveira et al. ${ }^{22}$ e Fávaro et al. ${ }^{23}$ utilizaram outras variáveis explanatórias. No estudo de Faváro et al..$^{23}$ o consumo alimentar inadequado das crianças representou um fator associado à insegurança alimentar. Dado indisponível no manuscrito ou análise não realizada.Tabela 3. Prevalências de insegurança alimentar,

doação de alimentos, em três ${ }^{11,25,26}$ de sete estu$\operatorname{dos}^{11,16,23-26,39}$ (42,8\% dos estudos) que mostraram famílias beneficiárias como as mais vulneráveis, foram outras variáveis que também apresentaram proporções expressivas de associação com a insegurança alimentar.

A escolaridade da mãe (quatro ${ }^{15,23,28,32}$ de 10 estudos $\left.^{12,14,15,21-23,28,31-33}\right)$; o sexo do chefe do domicílio (dois ${ }^{25,26}$ de seis estudos ${ }^{18,24,25,27,36,37}$ ); a idade da mãe (dois ${ }^{11,32}$ de seis estudos ${ }^{11,13,23,30,32,33}$ ); o local do domicílio (dois ${ }^{27,32}$ de 13 estu$\operatorname{dos}^{12,16-18,21,22,27-30,32,33,39}$ ); a coleta de lixo no domicílio ( um $^{35}$ de oito estudos ${ }^{15-17,19,35,39-41}$ ) e o abastecimento de água no domicílio ( $\mathrm{um}^{39}$ de nove estudos $^{15-17,19,26,35,39-41}$ ) foram outras variáveis que, também, mostraram-se associadas, nas análises bivariadas, à insegurança alimentar familiar.
O controle adequado das variáveis de confundimento foi conduzido em nove estu$\operatorname{dos}^{11,18,26,28,33,35,37-39}$. Considerando o conjunto desses estudos, a menor renda familiar ${ }^{11,26,35,37,39}$, a maior quantidade de indivíduos no domicílio ${ }^{18,35}$ e o tipo de moradia precária ${ }^{37,39}$ foram as condições que se associaram à insegurança alimentar familiar em no mínimo dois.

A Tabela 4 consolida os resultados encontrados para as categorias de investigações analisadas. Observa-se que a maior prevalência de IA foi encontrada entre as populações em iniquidades sociais $(87,2 \%)$. Da mesma forma apresentam-se os resultados relativos aos graus mais severos de IA, com prevalência de 35,8\% para a IAM e de $28,1 \%$ para a IAG ponderando as populações em iniquidades sociais. Assumindo-se a prevalência 
segundo estudos publicados no período de 2004-2013, envolvendo amostras representativas de cidades, regiões ou estados no Brasil.

\begin{tabular}{|c|c|c|c|c|c|c|c|}
\hline \multirow[t]{2}{*}{ Fonte } & \multirow[t]{2}{*}{$\begin{array}{l}\text { Local de } \\
\text { estudo }\end{array}$} & \multirow[t]{2}{*}{$\begin{array}{l}\text { Tamanho } \\
\text { amostral }\end{array}$} & \multicolumn{4}{|c|}{$\begin{array}{c}\text { Prevalências de } \\
\text { Insegurança Alimentar } \\
(\%)\end{array}$} & \multirow[t]{2}{*}{ Associações estatísticas } \\
\hline & & & IA & IAL & IAM & IAG & \\
\hline Lopes et al. ${ }^{25}$ & $\begin{array}{l}\text { Rio de } \\
\text { Janeiro (RJ) }\end{array}$ & 523 & 60,0 & 36,0 & - & - & $\begin{array}{l}\text { Análise bivariada } \\
\text { - Chefe do domicílio do sexo feminino } \\
\text { - Maior número de indivíduos no } \\
\text { domicílio - Presença de indivíduo menor } \\
\text { de cinco anos no domicílio } \\
\text { - Receber auxílio de programas de doação } \\
\text { de alimentos } \\
\text { - Renda familiar per capita inferior a US\$ } \\
60,00\end{array}$ \\
\hline $\begin{array}{l}\text { Guerra et } \\
\text { al. }^{26}\end{array}$ & $\begin{array}{l}\text { Alta Floresta, } \\
\text { Diamantino, } \\
\text { Sinop e } \\
\text { Sorriso } \\
\text { (MT) }\end{array}$ & 363 & 51,8 & 28,7 & 14,3 & 8,8 & $\begin{array}{l}\text { i) Análise bivariada } \\
\text { - Raça/cor preta dos adolescentes } \\
\text { - Escolaridade do chefe do domicílio entre } \\
\text { 0-4 anos } \\
\text { - Domicílio com } 7 \text { ou mais indivíduos } \\
\text { - Ausência de tratamento de água do } \\
\text { domicílio } \\
\text { - Participação em programa de } \\
\text { transferência de renda } \\
\text { - Renda familiar } \leq 1 / 2 \text { SM } \\
\text { ii) Regressão de Poisson } \\
\text { - Raça/cor preta do adolescente } \\
\text { - Chefe do domicílio com naturalidade } \\
\text { mato-grossense } \\
\text { - Ausência de tratamento de água no } \\
\text { domić́lio } \\
\text { - Renda familiar } \leq 1 \text { SM }\end{array}$ \\
\hline Rosa et al. ${ }^{27}$ & $\begin{array}{l}\text { Regiões do } \\
\text { Brasil } \\
\text { Domicílios } \\
\text { chefiados } \\
\text { por idosos } \\
\text { PNAD-2004 }\end{array}$ & 23877 & 29,8 & 12,7 & 11,5 & 5,6 & $\begin{array}{l}\text { Análise bivariada } \\
\text { - Domicílio chefiado por mulher } \\
\text { - Raça/cor indígena e preta/parda do chefe } \\
\text { do domicílio } \\
\text { - Menor escolaridade do chefe do } \\
\text { domicílio } \\
\text { - Maior número de indivíduos no } \\
\text { domicílio } \\
\text { - Região geográfica do Brasil onde se } \\
\text { localiza o domicílio (Norte e Nordeste) } \\
\text { - Domicílio localizado na zona rural } \\
\text { - Menor renda familiar per capita (SM) }\end{array}$ \\
\hline Kac et al. ${ }^{28}$ & $\begin{array}{l}\text { Regiões do } \\
\text { Brasil } \\
\text { Famílias com } \\
\text { crianças de } \\
0-60 \text { meses } \\
\text { PNDS-2006 }\end{array}$ & 3433 & 48,6 & - & - & - & $\begin{array}{l}\text { i) Análise bivariada } \\
\text { - Raça/cor preta ou marrom da mãe } \\
\text { - Menor escolaridade da mãe } \\
\text { - Região geográfica do Brasil onde se } \\
\text { localiza o domicílio (Norte) } \\
\text { - Maior número de crianças no domicílio } \\
\text { - Menor renda familiar (em quartis) } \\
\text { ii) Regressão linear } \\
\text { - Não foi encontrada associação }\end{array}$ \\
\hline
\end{tabular}

continua

de IA observada a partir dos estudos de base populacional de cidades, regiões ou estados no Bra- sil $(25,9 \%)$ como referência, verificou-se que a chance de uma família pertencente a populações 
Tabela 3. continuação

\begin{tabular}{|c|c|c|c|c|c|c|c|}
\hline \multirow[t]{2}{*}{ Fonte } & \multirow[t]{2}{*}{$\begin{array}{l}\text { Local de } \\
\text { estudo }\end{array}$} & \multirow[t]{2}{*}{$\begin{array}{l}\text { Tamanho } \\
\text { amostral }\end{array}$} & \multicolumn{4}{|c|}{$\begin{array}{c}\text { Prevalências de } \\
\text { Insegurança Alimentar } \\
(\%)\end{array}$} & \multirow[t]{2}{*}{ Associações estatísticas } \\
\hline & & & IA & IAL & IAM & IAG & \\
\hline Kac et al. ${ }^{29}$ & $\begin{array}{l}\text { Regiões do } \\
\text { Brasil } \\
\text { Famílias com } \\
\text { adolescentes } \\
\text { do sexo } \\
\text { feminino de } \\
\text { 15-18 anos } \\
\text { PNDS-2006 }\end{array}$ & 1529 & 40,8 & 26,6 & 9,4 & 4,8 & $\begin{array}{l}\text { Análise bivariada } \\
\text { - Idade do adolescente (15 anos) } \\
\text { - Raça/cor indígena e preta/parda do } \\
\text { adolescente } \\
\text { - Menor escolaridade do adolescente } \\
\text { (0-4anos) } \\
\text { - Região do Brasil onde se localiza o } \\
\text { domicílio (Nordeste) } \\
\text { - Maior número de indivíduos no } \\
\text { domicílio } \\
\text { - Menor renda familiar per capita (em } \\
\text { quartis) }\end{array}$ \\
\hline Tsai et al. ${ }^{30}$ & $\begin{array}{l}\text { Regiões do } \\
\text { Brasil } \\
\text { Mulheres } \\
\text { sexualmente } \\
\text { ativas } \\
\text { PNDS-2006 }\end{array}$ & 12684 & 23,8 & 13,9 & 6,2 & 3,7 & - Não foi encontrada associação \\
\hline $\begin{array}{l}\text { Gomes e } \\
\text { Gubert }^{31}\end{array}$ & $\begin{array}{l}\text { Regiões do } \\
\text { Brasil } \\
\text { Famílias } \\
\text { com crianças } \\
\text { menores de } \\
\text { dois anos } \\
\text { PNDS-2006 }\end{array}$ & 1635 & 47,2 & - & - & - & - Não foi encontrada associação \\
\hline $\begin{array}{l}\text { Velasquéz- } \\
\text { Melendez et } \\
\text { al. }{ }^{32}\end{array}$ & $\begin{array}{l}\text { Regiões do } \\
\text { Brasil } \\
\text { Mulheres } \\
\text { em idade } \\
\text { reprodutiva } \\
\text { e com filhos } \\
\text { menores de } \\
\text { cinco anos } \\
\text { PNDS-2006 }\end{array}$ & 10226 & 40,9 & 25,5 & 10,1 & 5,3 & $\begin{array}{l}\text { Análise bivariada } \\
\text { - Idade da mulher }<25 \text { anos } \\
\text { - Raça/cor preta ou marrom da mulher } \\
\text { - Menor escolaridade da mulher }(0-4 \\
\text { anos) } \\
\text { - Região geográfica do Brasil onde se } \\
\text { localiza o domicílio (Norte) } \\
\text { - Domicílio localizado na zona rural } \\
\text { - Menor renda familiar (em quartis) }\end{array}$ \\
\hline $\operatorname{Reis}^{33}$ & $\begin{array}{l}\text { Regiões do } \\
\text { Brasil } \\
\text { Famílias } \\
\text { com crianças } \\
\text { nascidas a } \\
\text { partir de } \\
\text { janeiro de } \\
2001 \\
\text { PNDS-2006 }\end{array}$ & 5000 & 35,8 & 23,0 & 8,0 & 4,8 & $\begin{array}{l}\text { Análise de Regressão } \\
\text { - Menor renda familiar per capita (em } \\
\text { reais) }\end{array}$ \\
\hline $\begin{array}{l}\text { Antunes et } \\
\text { al. }{ }^{34}\end{array}$ & $\begin{array}{l}\text { Duque de } \\
\text { Caxias (RJ) }\end{array}$ & 384 & 71,0 & 34,6 & - & - & - Não foi encontrada associação \\
\hline
\end{tabular}

continua

em iniquidades sociais estar em situação de insegurança alimentar foi 18,67 vezes maior (IC95\%: 8,68-42,89). Amostras provenientes de escolas/ creches e de serviços de saúde/beneficiários do Programa Bolsa Família também apresentaram maiores chances de IA, com valores de 4,61 
Tabela 3. continuação

\begin{tabular}{|c|c|c|c|c|c|c|c|}
\hline \multirow[t]{2}{*}{ Fonte } & \multirow[t]{2}{*}{$\begin{array}{l}\text { Local de } \\
\text { estudo }\end{array}$} & \multirow[t]{2}{*}{$\begin{array}{l}\text { Tamanho } \\
\text { amostral }\end{array}$} & \multicolumn{4}{|c|}{$\begin{array}{c}\text { Prevalências de } \\
\text { Insegurança Alimentar } \\
(\%)\end{array}$} & \multirow[t]{2}{*}{ Associações estatísticas } \\
\hline & & & IA & IAL & IAM & IAG & \\
\hline $\begin{array}{l}\text { Pimentel et } \\
\text { al. }{ }^{35}\end{array}$ & $\begin{array}{l}\text { Campos } \\
\text { Elíseos/ } \\
\text { Duque de } \\
\text { Caxias (RJ) }\end{array}$ & 402 & 72,0 & 39,0 & 21,0 & 12,0 & $\begin{array}{l}\text { i) Análise bivariada } \\
\text { - Menor escolaridade do chefe do } \\
\text { domicílio } \\
\text { - Maior número de indivíduos no } \\
\text { domicílio } \\
\text { - Ausência de coleta de lixo no domicílio } \\
\text { - Menor renda familiar per capita } \\
\text { - Classificações inferiores da ABIPEME } \\
\text { ii) Regressão logística multinomial } \\
\text { - Menor escolaridade do chefe do } \\
\text { domicílio } \\
\text { - Domicílio com } 5 \text { e mais indivíduos } \\
\text { - Ausência de filtro de água no domicílio } \\
\text { - Ausência de coleta de lixo no domicílio } \\
\text { - Menor renda familiar per capita (em } \\
\text { reais) } \\
\text { - Classificações inferiores da ABIPEME }\end{array}$ \\
\hline $\begin{array}{l}\text { Gubert e } \\
\text { Santos }^{36}\end{array}$ & $\begin{array}{l}\text { Distrito } \\
\text { Federal (DF) } \\
\text { PNAD-2004 }\end{array}$ & 621000 & 24,9 & 12,3 & 8,5 & 4,1 & $\begin{array}{l}\text { Análise bivariada } \\
\text { - Domicílios chefiados por idosos ou por } \\
\text { pessoas de raça/cor negra/parda } \\
\text { - Famílias com } 3 \text { ou mais crianças. } \\
\text { - Famílias com alta densidade } \\
\text { populacional por cômodo utilizado para } \\
\text { dormir } \\
\text { - Domicílios com renda familiar per capita } \\
\text { inferior a } 1 / 4 \text { de SM }\end{array}$ \\
\hline $\begin{array}{l}\text { Panigassi et } \\
\text { al. }{ }^{37}\end{array}$ & $\begin{array}{l}\text { Campinas } \\
\text { (SP) }\end{array}$ & 456 & 60,5 & 40,1 & - & - & $\begin{array}{l}\text { i) Análise bivariada } \\
\text { - Raça/cor preta ou parda do informante } \\
\text { - Esgotamento sanitário a céu aberto } \\
\text { ii) Regressão logística politômica } \\
\text { univariada } \\
\text { - Escolaridade do chefe abaixo do nível de } \\
\text { ensino fundamental } \\
\text { - Maior número de membros menores de } \\
5 \text { anos no domicílio } \\
\text { - Maior número de menores de } 18 \text { anos } \\
\text { no domicílio } \\
\text { - Família sem membro universitário } \\
\text { - Casa de alvenaria inacabada ou de } \\
\text { madeira/ zinco/papelão } \\
\text { - Renda familiar inferior a } 2 \text { SM } \\
\text { iii) Regressão logística politômica } \\
\text { - Maior número de pessoas por cômodo } \\
\text { - Casa de alvenaria inacabada ou de } \\
\text { madeira/zinco/papelão } \\
\text { - Renda familiar inferior a } 2 \text { SM }\end{array}$ \\
\hline
\end{tabular}

(IC95\%: 2,44-8,88) e 9,40 (IC95\%: 4,77-19,16), respectivamente. Resultado similar foi observado quando considerados como referência os resultados encontrados na PNAD. 
Tabela 3. continuação

\begin{tabular}{|c|c|c|c|c|c|c|c|}
\hline \multirow[t]{2}{*}{ Fonte } & \multirow[t]{2}{*}{$\begin{array}{l}\text { Local de } \\
\text { estudo }\end{array}$} & \multirow[t]{2}{*}{$\begin{array}{l}\text { Tamanho } \\
\text { amostral }\end{array}$} & \multicolumn{4}{|c|}{$\begin{array}{c}\text { Prevalências de } \\
\text { Insegurança Alimentar } \\
(\%)\end{array}$} & \multirow[t]{2}{*}{ Associações estatísticas } \\
\hline & & & IA & IAL & IAM & IAG & \\
\hline $\begin{array}{l}\text { Panigassi et } \\
\text { al. }{ }^{38}\end{array}$ & $\begin{array}{l}\text { Campinas } \\
\text { (SP) }\end{array}$ & 456 & 60,5 & 40,1 & 13,8 & 6,6 & $\begin{array}{l}\text { i) Análise bivariada } \\
\text { - Despesa familiar mensal com } \\
\text { alimentação (em reais) } \\
\text { ii) Regressão logística politônica } \\
\text { univariada } \\
\text { - Maior chance de IA para cada } 100 \text { reais a } \\
\text { menos gastos com a alimentação }\end{array}$ \\
\hline $\begin{array}{l}\text { Vianna } \\
\text { e Segall- } \\
\text { Corrêa }^{39}\end{array}$ & $\begin{array}{l}\text { Paraíba } \\
\text { (municípios } \\
\text { selecionados } \\
\text { para iniciar } \\
\text { o Programa } \\
\text { Fome Zero) }\end{array}$ & 4533 & 52,5 & 23,6 & 17,6 & 11,3 & $\begin{array}{l}\text { i) Análise bivariada } \\
\text { - Tipo de moradia precária } \\
\text { - Falta de acesso a serviços públicos } \\
\text { - Menor renda familiar per capita (em } \\
\text { reais) } \\
\text { ii) Regressão logística } \\
\text { - Tipo de moradia precária } \\
\text { - Falta de água permanente no domicílio } \\
\text { - Menor renda familiar per capita (em } \\
\text { reais) }\end{array}$ \\
\hline $\begin{array}{l}\text { Salles-Costa } \\
\text { et al. }{ }^{40}\end{array}$ & $\begin{array}{l}\text { Campos } \\
\text { Elíseos/ } \\
\text { Duque de } \\
\text { Caxias (RJ) }\end{array}$ & 1085 & 53,8 & 31,4 & 16,1 & 6,3 & $\begin{array}{l}\text { Análise bivariada } \\
\text { - Menor escolaridade do chefe do } \\
\text { domicílio } \\
\text { - Maior número de indivíduos no } \\
\text { domicílio } \\
\text { - Ausência de filtro de água no domicílio } \\
\text { - Menor renda familiar per capita (SM) } \\
\text { - Classificações inferiores da ABIPEME }\end{array}$ \\
\hline $\begin{array}{l}\text { Marín-Léon } \\
\text { et al. }{ }^{41}\end{array}$ & $\begin{array}{l}\text { Campinas } \\
\text { (SP) }\end{array}$ & 195 & 52,0 & 33,0 & 11,8 & 7,2 & $\begin{array}{l}\text { Análise bivariada } \\
\text { - Menor escolaridade do idoso do } \\
\text { domicílio } \\
\text { - Menor renda familiar (SM) }\end{array}$ \\
\hline \multicolumn{2}{|c|}{$\begin{array}{l}\text { Média ponderada pelo } \\
\text { tamanho amostral }\end{array}$} & 687781 & 25,9 & 12,9 & 8,6 & 4,2 & \\
\hline
\end{tabular}

IA: Insegurança Alimentar; IAL: Insegurança Alimentar Leve; IAM: Insegurança Alimentar Moderada; IAG: Insegurança Alimentar Grave; SM: Salário Mínimo; ABIPEME: Associação Brasileira de Institutos de Pesquisa de Mercado; PNAD: Pesquisa Nacional por Amostra de Domicílios; PNDS: Pesquisa Nacional de Demografia e Saúde.

Observações: Rosa et al. ${ }^{27}$, Kac et al. ${ }^{29}$, Tsai et al. ${ }^{30}$, Gomes et al. ${ }^{31}$, Velasquéz-Melendez et al. ${ }^{32}$, Antunes et al..$^{34}$, Pimentel et al..$^{35}$, Panigassi et al. ${ }^{37}$ e Marín-Léon et al. ${ }^{41}$ utilizaram outras variáveis explanatórias. Nesses estudos, a hospitalização da criança nos últimos 12 meses $^{27}$, o excesso de peso do adolescente ${ }^{29}$, o menor uso consistente de preservativo e a maior chance de corrimento vaginal na mulher ${ }^{30}$, a prática de aleitamento materno em crianças de 12- 24 meses $^{31}$, o hábito de fumar e a obesidade da mulher ${ }^{32}$, o menor consumo de energia, proteína e ferro pela criança ${ }^{34}$, as piores situações de estado nutricional infantil ${ }^{35} \mathrm{e}$ o consumo alimentar inadequado do informante ${ }^{38,41}$ representaram fatores associados à insegurança alimentar.

- : Dado indisponível no manuscrito ou análise não realizada.

No que se refere aos graus mais severos de insegurança alimentar, observou-se que a chance de uma família pertencente a populações em iniquidades sociais estar em IAM foi 5,64 (IC95\%: 2,45-14,26) e 8,72 vezes maior (IC95\%: 3,3926,82) quando comparadas às populações de base populacional de referência. Para os estudos de populações provenientes de serviços de saúde/ beneficiários do Programa Bolsa Família também se encontraram maiores chances de IAM em relação às bases populacionais. Observou-se que a chance de populações em iniquidades sociais estarem em situação de IAG foi 9,24 vezes maior (IC95\%: 3,04-37,86) quando comparadas às po- 
Tabela 4. Razões de Chances e Intervalos de Confiança de 95\% (IC95\%) de Insegurança Alimentar segundo diferentes categorias de amostras baseadas em estudos sobre segurança alimentar realizados no Brasil, no período de 2004 a 2013, assumindo como prevalências de referência as encontradas nos estudos representativos de cidades, regiões ou estados brasileiros e na PNAD.

\begin{tabular}{|c|c|c|c|c|c|}
\hline \multirow{3}{*}{ Proveniência da amostra } & \multirow{3}{*}{$\begin{array}{c}\text { Prevalências } \\
\text { de Insegurança } \\
\text { Alimentar }(\%) \\
\text { (Amplitude) }\end{array}$} & \multicolumn{4}{|c|}{ Referência } \\
\hline & & \multicolumn{2}{|c|}{$\begin{array}{l}\text { Base populacional do } \\
\text { Brasil (PNAD, 2009) }\end{array}$} & \multicolumn{2}{|c|}{$\begin{array}{l}\text { Base populacional de } \\
\text { cidades, regiões ou } \\
\text { estados no Brasil }\end{array}$} \\
\hline & & $\begin{array}{l}\text { Razão de } \\
\text { Chances } \\
\text { (IC95\%) }\end{array}$ & $\begin{array}{l}\text { Valor } \\
\text { de p } \\
\left(X^{2}\right)\end{array}$ & $\begin{array}{l}\text { Razão de } \\
\text { Chances } \\
\text { (IC95\%) }\end{array}$ & $\begin{array}{l}\text { Valor } \\
\text { de p } \\
\left(X^{2}\right)\end{array}$ \\
\hline \multicolumn{6}{|l|}{ Insegurança Alimentar } \\
\hline Base populacional do Brasil (PNAD, 2009) & 30,2 & 1 & & - & - \\
\hline $\begin{array}{l}\text { Base populacional de cidades, regiões ou } \\
\text { estados no Brasil }\end{array}$ & $25,9(72,0-23,8=48,2)$ & - & - & 1 & \\
\hline Escolas/creches & $61,8(64,5-46,6=17,9)$ & $3,78(2,03-7,16)$ & $<0,001^{*}$ & $4,61(2,44-8,88)$ & $<0,001^{*}$ \\
\hline Populações em iniquidades sociais & $87,2(88,2-75,5=12,7)$ & $15,34(7,22-34,78)$ & $<0,001^{*}$ & $18,67(8,68-42,89)$ & $<0,001^{*}$ \\
\hline $\begin{array}{l}\text { Serviços de saúde/beneficiários do } \\
\text { Programa Bolsa Família }\end{array}$ & $76,6(88,0-72,0=16,0)$ & $7,72(3,97-15,47)$ & $<0,001^{*}$ & $9,40(4,77-19,16)$ & $<0,001^{*}$ \\
\hline \multicolumn{6}{|l|}{ Insegurança Alimentar Leve } \\
\hline Base populacional do Brasil (PNAD, 2009) & 18,7 & 1 & & - & - \\
\hline $\begin{array}{l}\text { Base populacional de cidades, regiões ou } \\
\text { estados no Brasil }\end{array}$ & $12,9(40,1-12,3=27,8)$ & - & - & 1 & \\
\hline Escolas/creches & $39,5(42,5-32,4=10,1)$ & $2,83(1,44-5,72)$ & $<0,001^{*}$ & $4,42(2,11-9,83)$ & $<0,001^{*}$ \\
\hline Populações em iniquidades sociais & $23,2(44,6-17,8=26,8)$ & $1,27(0,61-2,68)$ & 0,603 & $1,99(0,90-4,60)$ & 0,096 \\
\hline $\begin{array}{l}\text { Serviços de saúde/beneficiários do } \\
\text { Programa Bolsa Família }\end{array}$ & $45,0(50,4-35,0=15,4)$ & $3,46(1,77-6,98)$ & $<0,001^{*}$ & $5,43(2,59-12,01)$ & $<0,001^{*}$ \\
\hline \multicolumn{6}{|l|}{ Insegurança Alimentar Moderada } \\
\hline Base populacional do Brasil (PNAD, 2009) & 6,5 & 1 & & - & - \\
\hline $\begin{array}{l}\text { Base populacional de cidades, regiões ou } \\
\text { estados no Brasil }\end{array}$ & $8,6(21,0-6,2=14,8)$ & - & - & 1 & \\
\hline Escolas/creches & $16,2(18,0-15,9=2,1)$ & $2,97(1,05-9,70)$ & $0,040^{*}$ & $1,92(0,75-5,21)$ & 0,198 \\
\hline Populações em iniquidades sociais & $35,8(40,2-27,7=12,5)$ & $8,72(3,39-26,82)$ & $<0,001^{*}$ & $5,64(2,45-14,26)$ & $<0,001^{*}$ \\
\hline $\begin{array}{l}\text { Serviços de saúde/beneficiários do } \\
\text { Programa Bolsa Família }\end{array}$ & $20,3(28,5-10,7=17,8)$ & $3,89(1,42-12,43)$ & $0,005^{*}$ & $2,52(1,03-6,65)$ & $0,043^{*}$ \\
\hline \multicolumn{6}{|l|}{ Insegurança Alimentar Grave } \\
\hline Base populacional do Brasil (PNAD, 2009) & 5,0 & 1 & & - & - \\
\hline $\begin{array}{l}\text { Base populacional de cidades, regiões ou } \\
\text { estados no Brasil }\end{array}$ & $4,2(12,0-3,7=8,3)$ & - & - & 1 & \\
\hline Escolas/creches & $7,5(11,7-6,1=5,6)$ & $1,65(0,45-6,65)$ & 0,567 & $2,08(0,53-9,76)$ & 0,373 \\
\hline Populações em iniquidades sociais & $28,1(36,9-15,7=21,2)$ & $7,32(2,62-25,48)$ & $<0,001^{*}$ & $9,24(3,04-37,86)$ & $<0,001^{*}$ \\
\hline $\begin{array}{l}\text { Serviços de saúde/beneficiários do } \\
\text { Programa Bolsa Família }\end{array}$ & $11,3(24,5-5,9=18,6)$ & $2,34(0,72-8,94)$ & 0,191 & $2,95(0,84-13,17)$ & 0,104 \\
\hline
\end{tabular}

*Diferença estatisticamente significativa em relação à prevalência estimada a partir de estudos representativos de cidades, regiões ou estados no Brasil e da PNAD, 2009. PNAD: Pesquisa Nacional por Amostra de Domicílios.

pulações de base populacional de cidades, regiões ou estados no Brasil e 7,32 vezes maior (IC95\%: 2,62-25,48) em relação aos resultados da PNAD.

\section{Discussão}

Nas últimas décadas, em várias partes do mundo, observou-se um aumento significativo das investigações sobre Segurança Alimentar, abordando tanto o diagnóstico das populações vulneráveis à fome como seus determinantes e consequên- 
cias para a sua saúde e bem estar ${ }^{18}$. No Brasil, são vários os grupos de pesquisa que se preocupam com o tema. Dentre eles, destacam-se os que realizam estudos sobre a qualidade do alimento e sobre o estado nutricional de grupos populacionais. Entretanto, sugere-se ainda que as pesquisas sobre SAN no país precisam de maior dinamismo e integração entre os focos econômico e social ${ }^{42}$.

A insegurança alimentar tem sido considerada como um problema de saúde mundial ${ }^{26}$. Nos Estados Unidos, uma pesquisa envolvendo crianças e famílias de baixa renda, encontrou $30,7 \%$ de insegurança alimentar ${ }^{43}$. Na Colômbia, um estudo também conduzido com famílias de baixa renda observou 51,8\% de insegurança alimentar ${ }^{44}$. Em estudo realizado envolvendo famílias com crianças de Quebec, no Canadá, e da Jamaica observaram-se prevalências de insegurança alimentar de 9,0\% e $26,0 \%$, respectivamente $^{45}$. No Brasil, a PNAD ${ }^{10}$ identificou que $30,2 \%$ da população apresentava algum grau de insegurança alimentar. Por meio dos resultados sistematizados no presente trabalho, ressalta-se que a condição das famílias de amostras provenientes de escolas/creches, de serviços de saúde/ beneficiários do Programa Bolsa Família e de caracterização em iniquidade social é pior à de outros países ${ }^{43-45}$ e do Brasil $^{10}$. Esses achados são alarmantes e evidenciam que, apesar dos avanços observados na diminuição das desigualdades no Brasil, ainda é grande o contingente de pessoas que vivem em situação de insegurança alimentar, com desigualdades regionais e de outras índoles como de cor/raça, faixa etária, faixa de renda e localização urbano vs. rural, que implicam em importantes desafios ${ }^{46}$.

$\mathrm{Na}$ linha de pensamento anterior, tomando como exemplo a média ponderada de insegurança alimentar para os estudos com amostras obtidas em populações em iniquidades sociais $(87,2 \%)$, que podemos considerar muito alta, deve ser reforçada a questão do acesso inadequado e insuficiente aos alimentos como consequência, principalmente, das desigualdades socioeconômicas existentes na sociedade brasileira ${ }^{15}$. Análise similar deriva das outras categorias consideradas que, também, podem ser caracterizadas pela vulnerabilidade socioeconômica ao considerar que tanto os beneficiários de escolas/creches públicas ${ }^{20}$ quanto os dos serviços públicos de saúde/Programa Bolsa Família ${ }^{47}$ representam classes sociais inferiores. No sentido específico dos programas de transferência de renda, revisão recente da literatura destacou a impossibilidade desses programas, apenas com as suas ações, re- solverem o problema da pobreza e da insegurança alimentar ${ }^{48}$.

As populações em iniquidades sociais do presente estudo, assumidas como as de maior grau de pobreza, apresentaram maiores chances de insegurança alimentar moderada e grave, justificando o contexto de vulnerabilidades do ponto de vista da segurança alimentar e nutricional (acesso a terra, água, bens e serviços públicos, condições dignas de moradia e consumo de alimentos) associado à pobreza ${ }^{47}$. Estudos de abrangência nacional ${ }^{10,49}$, semelhantemente, têm indicado maior prevalência de insegurança alimentar moderada e grave entre as famílias mais pobres.

A insegurança alimentar é reconhecida como um fenômeno complexo e multidimensional de determinação multicausal ${ }^{36,50,51}$. Nesse sentido, os fatores que determinam a situação alimentar de uma população apresentam naturezas distintas, entre as quais a econômica, a psicossocial, a ética, a política e a cultural ${ }^{52}$. No presente trabalho, a renda familiar foi a variável que se apresentou em maior quantidade de vezes associada significativamente à insegurança alimentar entre os estudos analisados. De maneira coerente, os resultados da $\mathrm{PNAD}^{10}$ evidenciaram que quanto menor for o rendimento mensal domiciliar per capita, maior a proporção de domicílios com insegurança alimentar. Esses resultados apresentam ainda maior relevância apreciando as evidências sobre a proporção significativa da renda total que é utilizada para a alimentação ${ }^{53}$. Do ponto de vista metodológico, esses achados também são relevantes, pois destacam a utilidade da medição da insegurança alimentar por meio da EBIA para o monitoramento da iniquidade, podendo complementar um conjunto de indicadores sociais ou, mesmo de forma isolada, identificar grupos com vulnerabilidade social, como relatado anteriomente ${ }^{37}$.

A contribuição da quantidade de indivíduos no domicílio na insegurança alimentar explicada em estudos brasileiros de âmbito nacional ${ }^{8,54}$ foi, igualmente, sistematizada no presente trabalho. Essa relação pode ser explicada pelo fato de famílias grandes necessitarem de mais recursos para a compra de alimentos, o que normalmente não acompanha o crescimento familiar ${ }^{18}$.

Entre os estudos analisados, o tipo de moradia foi a outra condição que mostrou com maior força sua importância na determinação da insegurança alimentar, o que pode estar atrelado aos fatores socioeconômicos e aos determinantes demográficos que influenciam esta condição ${ }^{37}$. Neste sentido, deve-se ressaltar, ainda, que as moradias inacabadas proporcionam condições de insalubridade 
aos seus ocupantes e favorecem o surgimento de doenças entre os indivíduos mais vulneráveis ${ }^{55}$. A qualidade da habitação relaciona-se com a segurança alimentar na medida em que contribui para o aproveitamento biológico do alimento, evitando a proliferação de inúmeras doenças ${ }^{56}$.

Os resultados do presente estudo somam-se aos de revisão anterior que sistematizou a relação da insegurança alimentar com indicadores sociais, como menor renda e escolaridade, ausência de vínculo empregatício e saneamento básico ${ }^{7}$. Esses fatores representam, também, as principais condições relacionadas com problemas de crescimento linear entre as crianças brasileiras menores de cinco anos, numa conjuntura na qual esses dois eventos, apesar de distintos, apresentam-se associados e com determinação social ${ }^{8}$.

Com base nos dados aqui analisados, considerando tanto as frequências de insegurança alimentar nos diferentes cenários sociodemográficos analisados, quanto os fatores associados, evidencia-se, claramente, a determinação social da insegurança alimentar no Brasil. Devido à composição da insegurança alimentar como problema de grave significância populacional e de conotação socioeconômica, reforça-se a atenção prioritária que deve merecer por parte das diferentes instâncias governamentais do país, com a efetivação de políticas públicas de combate à fome e à miséria.

\section{Colaboradores}

TA Bezerra participou da análise e interpretação dos dados, redação, correção e revisão crítica do artigo. RA Olinda participou da análise e interpretação dos dados, correção e revisão crítica do artigo. DF Pedraza participou da concepção do estudo, análise e interpretação dos dados, redação, correção e revisão crítica do artigo. 


\section{Referências}

1. Brasil. II Conferência Nacional de Segurança Alimentar e Nutricional: A construção da Política Nacional de Segurança Alimentar. Relatório Final. 2004. [acessado: 2012 jul 20]. Disponível em: http://www.fomezero.gov.br

2. Nascimento AL, Andrade SLLS. Segurança alimentar e nutricional: pressupostos para uma nova cidadania? Ciênc Cult 2010; 62(4):34-38.

3. Pereira RA, Santos LMP. A dimensão da insegurança alimentar. Rev Nutr 2008; 21(Supl):S7-S13.

4. Pérez-Escamilla R, Segall-Corrêa AM. Food insecurity measurement and indicators. Rev Nutr 2008; 21(Supl.): 15-26.

5. Segall-Corrêa AM, Marín-León L. Segurança Alimentar no Brasil: Proposição e Usos da Escala Brasileira de Medida da Insegurança Alimentar (EBIA) de 2003 a 2009. Segur Aliment e Nutr 2009; 16(2):1-19.

6. Nobre LN, Murta NMG, Souza MM, Ferreira NC, Cardoso LM, Hamacek FR. Segurança Alimentar em uma Comunidade Rural no Alto Vale do Jequitinhonha/ MG. Segur Aliment e Nutr 2009; 16(1):18-31.

7. Morais DC, Dutra LV, Franceschini SCC, Priore SE Insegurança alimentar e indicadores antropométricos, dietéticos e sociais em estudos brasileiros: uma revisão sistemática. Cien Saude Colet 2014; 19(5):1475-1488.

8. Figueroa Pedraza D. Crescimento linear das crianças brasileiras: reflexões no contexto da equidade social. Rev Nutr 2016; 29(2):287-296

9. Kepple AW, Segall-Corrêa AM. Conceituando e medindo segurança alimentar e nutricional. Cien Saude Colet 2011; 16(1):187-199.

10. Instituto Brasileiro de Geografia e Estatística (IBGE). Pesquisa Nacional por Amostra de Domicílios. Segurança Alimentar 2009. Rio de Janeiro: IBGE; 2009.

11. Figueroa Pedraza D, Queiroz D, Menezes TN. Segurança alimentar em famílias com crianças matriculadas em creches públicas do Estado da Paraíba, Brasil. Rev Nutr 2013; 26(5):517-527.

12. Ribeiro-Silva RC, Oliveira-Assis $A M$, Junqueira $A B$ Fiaccone RL, Santos SMC, Barreto ML, Jesus Pinto E, Silva LA, Rodrigues LC, Alcantara-Neves NM. Food insecurity. Food and nutrition insecurity: a marker of vulnerability to asthma symptoms. Public Health Nutr 2012; 20(1):1-6.

13. Souza MM, Figueroa Pedraza D, Menezes TN. Estado Nutricional de crianças assistidas em creches e situação de (in)segurança alimentar de suas famílias. Cien Saude Colet 2012; 17(12):3425-3436.

14. Vieira VL, Souza JMP, Cervato-Mancuso AM. Insegurança alimentar, vínculo mãe-filho e desnutrição infantil em área de alta vulnerabilidade social. Rev Bras Saúde Matern Infant 2010; 10(2):199-207.

15. Souza NN, Dias MM, Sperandio N, Franceschini SCC Priore SE. Perfil socioeconômico e insegurança alimentar e nutricional de famílias beneficiárias do Programa Bolsa Família no município de Viçosa, Estado de Minas Gerais, Brasil, em 2011: um estudo epidemiológico transversal. Epidemiol Serv Saúde 2012; 21(4):655-662.

16. Silva CCS, Oliveira KBB, Alves AS, Neves JA, Modesto CAC, Vianna RRT. Associação entre consumo alimentar e (in)segurança alimentar e nutricional em São José dos Ramos-PB. Braz J Food Technol 2012; 15(n esp.):23-30.
17. Dias MM, Machado HM, Ferreira CS, Oliveira VL, Gomes AP, Cantaluppi ETC. Situação de insegurança alimentar de famílias beneficiárias de programas de transferência de renda na Unidade de Saúde da Família Comunidade São João Baptista, Petropólis, Rio de Janeiro. Rev APS 2012; 15(2):199-205.

18. Anschau FR, Matshuo T, Segall-Corrêa AM. Insegurança alimentar entre beneficiários de programas de transferência de renda. Rev Nutr 2012; 25(2):177-189.

19. Magrani LHA, Paula MELS, Coutinho BBP, Guerra DM, Tavares RM, Silveira MO. Insegurança alimentar e estado nutricional das famílias que recebem benefícios sociais na Unidade de Saúde da Família Vila Felipe Petropólis - RJ. Rev APS 2012; 15(1):29-35.

20. Aires JS, Martins MC, Joventino ES, Ximenes LB. (In)segurança alimentar em famílias de pré-escolares de uma zona rural do Ceará. Acta Paul Enferm 2012; 25(1):102-108.

21. Oliveira JS, Lira PIC, Maia SR, Sequeira LAS, Amorim RCA, Batista Filho M. Insegurança alimentar e estado nutricional de crianças de Gameleira, zona da mata do Nordeste brasileiro. Rev Bras Saúde Matern Infant 2010; 10(2):237-245

22. Oliveira JS, Lira PIC, Andrade ALLS, Sales AC, Maia SR, Batista Filho M. Insegurança alimentar e estado nutricional de crianças de São João do Tigre, no semi-árido do Nordeste. Rev Bras Epidemiol 2009; 12(3):413-423.

23. Fávaro T, Ribas DLB, Zorzatto JR, Segall-Corrêa AM Panigassi G. Segurança alimentar em famílias indígenas Teréna, Mato Grosso do Sul, Brasil. Cad Saude Publica 2007; 23(4):785-793.

24. Pereira DA, Vieira VL, Fiore EG, Cervato-Mancurso AM. Insegurança alimentar em região de alta vulnerabilidade social da cidade São Paulo. Segur Aliment e Nutr 2006; 13(2):34-42.

25. Lopes TS, Sichieri R, Salles-Costa R, Veiga GV, Pereira RA. Family food insecurity and nutritional risk in adolescents from a low-income area of Rio de Janeiro, Brazil. J Biosoc Sci 2013; 45(5):661-674.

26. Guerra LDS, Espinosa MM, Bezerra ACD, Guimarães LV, Lima-Lopes MA. Insegurança alimentar em domić́lios da Amazônia Legal Brasileira: prevalência e fatores associados. Cad Saude Publica 2013; 29(2):335-348.

27. Rosa TEC, Mondini L, Gubert MB, Sato GS, Benício MHA. Segurança alimentar em domicílios chefiados por idosos, Brasil. Rev Bras Geriatr Gerontol 2012; 15(1):69-77.

28. Kac G, Schüssel MM, Pérez-Escamilla, Velásquez-Melendez G, Silva AAM. Household food insecurity is not associated with BMI for age or weighr for height among Brazilian children aged 0-60 months. Plos One 2012; 7:e45747.

29. Kac G, Velásquez-Meléndez G, Schlüssel MM, Segall-Corrêa AM, Silva AA, Pérez-Escamilla R. Severe food insecurity is associated with obesity among Brazilian adolescent females. Public Health Nutr. 2012; 15(10):1854-1860

30. Tsai AC, Hung KJ, Weiser SD. Is food insecurity with HIV risk? Cross-sectional evidence from sexually active women in Brazil. Plos Med. 2012; 9(4):e10001203. 
31. Gomes FP, Gubert MB. Breastfeeding in children under 2 years old and household food nutrition security status. J Pediatr 2012; 88(3):279-82.

32. Velásquez-Meléndez G, Schlüssel MM, Brito AS, Silva AA, Lopes-Filho JD, Kac G. Mild but not light or severe food insecurity is associated with obesity among Brazilian women. J. Nutr. 2011; 141(5):898-902.

33. Reis M. Food insecurity and the relationship between household income and children's health and nutrition in Brazil. Health Econ. 2012; 21(4):405-427.

34. Antunes MML, Sichieri R, Salles-Costa R. Consumo alimentar de crianças menores de três anos residentes em área de alta prevalência de insegurança alimentar domiciliar. Cad Saude Publica 2010; 26(8):1642-50.

35. Pimentel PG, Sicheri R, Salles-Costa R. Insegurança alimentar, condições socioeconômicas e indicadores antropométricos em crianças da Região Metropolitana do Rio de Janeiro, Brasil. Rev Bras Estud Popul. 2009; 26(2):283-94.

36. Gubert MB, Santos LMP. Determinantes da insegurança alimentar no Distrito Federal. Comun Ciênc Saúde 2009; 20(2):143-50.

37. Panigassi G, Segall-Corrêa AM, Marín-Léon L, Pérez-Escamilla R, Sampaio MFA, Maranha LKA. Insegurança alimentar como indicador de iniquidade: análise de inquérito populacional. Cad Saude Publica 2008; 24(10):2376-2384.

38. Panigassi G, Segall-Corrêa AM, Marín-Léon L, Pérez-Escamilla R, Maranha LKA, Sampaio MFA. Insegurança alimentar intrafamiliar e perfil de consumo de alimentos. Rev Nutr 2008; 21(Supl):135-144.

39. Vianna RPT, Segall-Corrêa AM. Insegurança alimentar das famílias residentes em municípios do interior do estado da Paraíba, Brasil. Rev Nutr 2008; 21(Supl):11122.

40. Salles-Costa R, Pereira RA, Vasconcellos MTL, Veiga GV, Marins VMR, Jardim BC, Gomes FS, Sichieri R. Associação entre fatores socioeconômicos e insegurança alimentar: estudo de base populacional na Região Metropolitana do Rio de Janeiro, Brasil. Rev Nutr 2008; 21(Supl.):99-109.

41. Marín-Léon L, Segall-Corrêa, Panigassi G, Maranha LK, Sampaio MFA, Pérez-Escamilla R. A percepção de insegurança alimentar em famílias com idosos em Campinas São Paulo, Brasil. Cad Saude Publica 2005; 21(5):1433-1440.

42. Prado SD, Gugelmin SA, Mattos RA, Silva JK, Olivares PSG. A pesquisa sobre segurança alimentar e nutricional no Brasil de 2000 a 2005: tendências e desafios. Cien Saude Colet 2010; 15(1):7-18.

43. Mettalinos-Katsaras E, Sherry B, Kallio J. Food insecurity is associated with owerweight in children younger than 5 years of age. J Am Diet Assoc. 2009; 109(10):1790-4.

44. Hackett M, Melgar-Quinonez H, Taylor CA, Uribe MCA. Factors associated with household food security of participants of the MANA food supplement program in Colombia. Arch Latinoam Nutr 2010; (60)1:4247.
45. Dubois L, Francis D, Burnier D, Tatone-Tokuda F, Girard M, Gordon-Strachan G, Fox K, Wilks R. Household food insecurity and childhood overweight in Jamaica and Québec: a gender-based analysis. BMC Public Health 2011; 11(199):1-10.

46. Kepple AW. O estado da segurança alimentar e nutricional no Brasil: Um retrato multidimensional: Relatório 2014. Brasília: FAO; 2014.

47. Traldi DRC, Almeida LLMC. Políticas públicas de transferência de renda e a questão da segurança alimentar dos beneficiários: efetividades e entraves do Programa Bolsa Família. Politica \& Sociedades 2012; 11(21):137-171.

48. Cotta RMM, Machado JC. Programa Bolsa Família e segurança alimentar e nutricional no Brasil: revisão crítica da literatura. Rev Panam Salud Pública 2013; 33(1):54-60.

49. Brasil. Ministério da Saúde (MS). Centro Brasileiro de Análise e Planejamento. Pesquisa Nacional de Demografia e Saúde da Mulher-PNDS: 2006: dimensões do processo reprodutivo e da Saúde da Criança. Brasília: MS; 2009.

50. Melgar-Quinonez H, Hackett M. Measuring household food security: the global experience. Rev Nutr 2008; 21(Supl.):27-37.

51. Albuquerque MFM. A segurança alimentar e nutricional e o uso da abordagem de direitos humanos no desenho das políticas públicas para combater a fome e a pobreza. Rev Nutr 2009; 22(6):895-903.

52. Ação Brasileira pela Nutrição e Direitos Humanos (ABRANDH). O direito humano à alimentação adequada e o sistema nacional de segurança alimentar e nutricional. Brasília: ABRANDH; 2013.

53. Instituto Brasileiro de Análises Sociais e Econômicas (Ibase). Repercussões do Programa Bolsa Família na segurança alimentar e nutricional das famílias beneficiadas. Rio de Janeiro: Ibase; 2008.

54. Hoffman R. Determinantes da Insegurança Alimentar no Brasil: Análise dos Dados da PNAD de 2004. Segur Aliment e Nutr 2008; 15(1):49-61.

55. Burity V, Franceschini T, Valente F, Recine E, Leão M, Carvalho MF. Direito Humano à Alimentação Adequada no Contexto da Segurança Alimentar e Nutricional. Brasília: ABRANDH; 2010.

56. Carvalho Filho EV, Aquino JS, Vianna RPT. Insegurança e Consumo Alimentar no Município de Princesa Isabel - Paraíba - Brasil. Rev Brasileira de Ciências da Saúde 2006; 10(3):277-286.

Artigo apresentado em 23/07/2015

Aprovado em 13/01/2016

Versão final apresentada em 15/01/2016 
